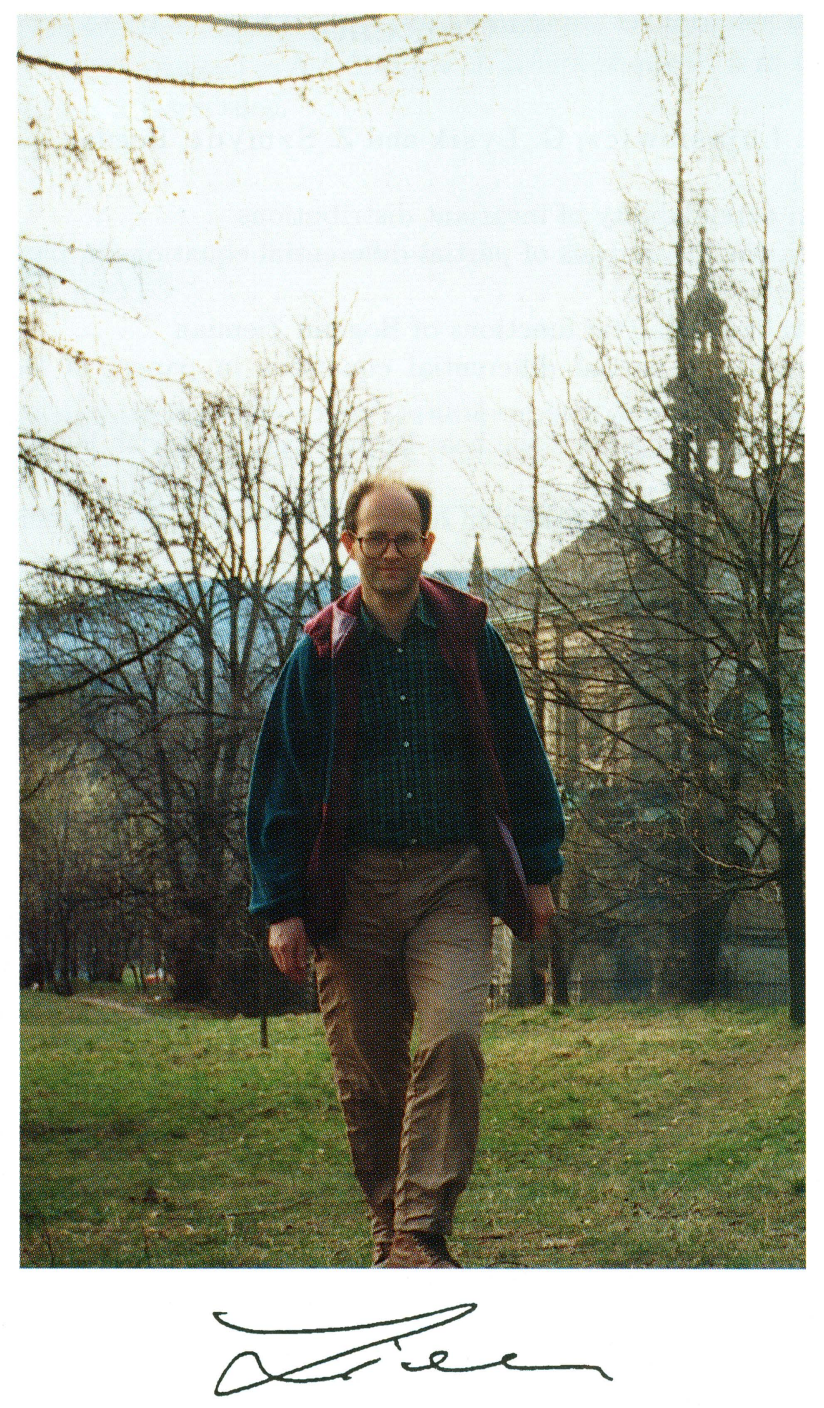




\title{
Bogdan Ziemian (1953-1997)
}

\author{
by Bogdan Bojarski (Warszawa), StanisŁaW Łojasiewicz (Kraków), \\ Grzegorz Łysik (Warszawa) and Zofia SzMydt (Warszawa**
}

Bogdan Ziemian was born on April 28, 1953 in Jelenia Góra. He studied at the Department of Mathematics, Computer Sciences and Mechanics of Warsaw University in 1972-1976. He began his work at the Institute of Mathematics of the Polish Academy of Sciences (IM PAS) in 1976 and worked there till the day he died. He obtained his Ph.D. degree there in 1981 under the supervision of Zofia Szmydt. He was promoted to associate professor in 1986 and to full professorship in 1993.

The mainstream of Bogdan Ziemian's research was focused on differential equations. He introduced new original methods in this field, based on a widely understood mathematical analysis: Lie groups, complex analysis, functional analysis, distributions and hyperfunctions.

Bogdan Ziemian was gifted with great analytical and geometrical intuition and imagination. His ability to see known facts from entirely new perspectives resulted in many surprising, but at the same time fruitful and simple ideas. Discovering links between mathematical theories which used to be treated separately is one of the most characteristic features of his research.

Much of his work, important for the theory of distributions and integral transformations, was devoted to creating a mathematical apparatus to study singular partial differential equations, the principal focus of his research. Developing these tools often required overcoming considerable technical difficulties. This is particularly evident in the papers [12-14], and in closely related work [19, 24].

* The authors would like to thank the colleagues of Bogdan Ziemian for their remarks that helped to improve the text. 
In the following, we treat chronologically the main stages of Ziemian's scientific work. We also refer to notes [R1]-[R3] in this volume, devoted to characterization of some groups of his papers.

B. Ziemian worked under Zofia Szmydt's guidance from his third year of university studies and very early began to present his own results. His first papers $[1,2,4,6]$ and $[46]\left({ }^{1}\right)$ concerned the study of distributions invariant under an action of a connected Lie group $G$ of (local) diffeomorphisms of a manifold $M$. In [4], $G$-invariant distributions on $M$ on which $G$ acts smoothly are characterized in terms of suitably defined distributions on the orbit space $M / G$. Since $M / G$ need not be Hausdorff, the construction of distributions on it is not trivial, and requires the so-called Hausdorff partition. As a consequence of that characterization, B. Ziemian proved that every $G$ invariant distribution $u$ is a weak limit of $G$-invariant smooth functions, and it can be represented in the form $u=P f$, where $P$ is a $C^{\infty}$ locally invariant differential operator, and $f$ is a $G$-invariant continuous function. Another description of distributions invariant under a compact Lie group was obtained in [6] by introducing the concept of an elliptic space defined with the help of invariant elliptic operators. This concept also allowed Ziemian to relate singularities of the orbit space to the loss of ellipticity of an elliptic operator on the boundary of the space. In [46], which together with [4] and [5] constituted his doctoral thesis, he studied the problem of extension of a distribution invariant under a Lie group from the set of orbits of maximal dimension to the whole space.

The paper [5] is one in a series $[3,5,7-10,15,16,18]$ (written jointly with Z. Szmydt) devoted to linear differential operators $P$ with polynomial coefficients, and invariant with respect to the form $S_{p, q}=\sum_{i=1}^{n} x_{i}^{p}-\sum_{i=1}^{m} y_{i}^{q}$. The method applied in [5] to find solutions of the equations $P u=\delta$ and $P u=0$ is a refinement of Garding's method used for the ultrahyperbolic operator $\square$, which transforms the problem to the 1-dimensional case by applying the operation $K$ of averaging smooth functions over the hypersurfaces $S_{p, q}(x, y)=s_{0}$. The methods of Gårding and Tengstrand used for the $\square$ operator are not applicable in this more general case. The main problem lies in establishing smoothness properties of the operation $K$. This was done by applying suitable singular partitions of unity. This method allowed effective calculations of fundamental solutions of classical invariant operators.

In [10], a differential operator $P$ on a real analytic manifold $X$ is assumed to be invariant with respect to a real analytic function $F$ on $X$, i.e., $P(f \circ F)=Q f \circ F$ for any $f \in C^{\infty}(F(X))$, where $Q$ is an ordinary differential operator with analytic coefficients. Then, for any isolated critical

$\left({ }^{1}\right)$ The manuscript of [46] was written by Ziemian in 1981 as a part of his doctoral thesis at IM PAS. 
point $z$ of $F$, and for any $F$-invariant distribution $v$ on $X$, local solutions to $P u=v$ are found near $z$ and have the form of a series of $F$-invariant distributions.

The scientific interests of Ziemian evolved later into microlocal analysis. The starting point here was his habilitation thesis [12-14], followed by its enlarged version [23]. The thesis was devoted to distributions on the real line, for which he established a generalized Taylor decomposition. The idea of the generalization comes from interpretation of the Taylor formula as a spectral decomposition of a function with respect to the functions $x^{\alpha}, \alpha \in \mathbb{C}$ (i.e. the eigenfunctions of the operator $x d / d x$ ), modulo flat functions. In the case of a function $u$ smooth at zero, this spectral decomposition takes the form

$$
u(x)=S^{r}\left[x^{\alpha}\right]+R^{r}(x) \quad \text { for small } x>0,
$$

where $S^{r}=\sum_{i=0}^{r-1} \frac{u^{i}(0)}{i !} \delta_{(i)}$ is a distribution acting on the function $\alpha \mapsto x^{\alpha}$ with fixed $x>0$, and the rest $R^{r}$ is $r$-flat at zero (i.e. $R^{r} \in O\left(x^{r}\right)$ ). In general, if $u$ is a measurable function on $(0, \varrho)$ and $u \in O\left(x^{m}\right)$ for some $m \in \mathbb{R}$, or $u$ is a distribution on $[0, \varrho)$ of order $-m$ for some $m \in-\mathbb{N}_{0}$, then it is necessary to take a spectral hyperfunction carried by $\{z \in \mathbb{C}$ : $|z-m| \leq r\} \cap \operatorname{Re} z \geq m\}$ for $S^{r}$ in $(*)$; the flatness of the rest is measured in terms of holomorphicity of its Mellin transformation. This connection discovered between the theory of the Mellin transformation and the theory of hyperfunctions enabled Ziemian to capture a new image of the notion of differentiability [14]; its application to the study of singularities of solutions to linear ordinary differential equations with smooth coefficients appeared in [13]. Let us quote here the first impression of H. Komatsu about these papers: "I started to read his papers only recently and was fascinated at once". A similar impression was expressed by the late A. Pliś.

The extension of these results to the $n$-dimensional case $[19,24]$ required the development of multidimensional Mellin analysis, defining a class of distributions admitting a generalized Taylor decomposition, and operating with methods of hyperfunctions in several variables; for instance, in [30], Ziemian originated the modified Cauchy transformation. The theory of multidimensional Mellin transformation was then applied jointly with Z. Szmydt, in $[20,22,25]$, to the study of the equation $R u=f$, where $R$ is an elliptic Fuchsian type operator of order $m$ with smooth coefficients in a neighbourhood of zero. The papers concern the existence of solutions having a given regularity near the vertex 0 of the $n$-dimensional cone $\mathbb{R}_{+}^{n}:=\left(\mathbb{R}_{+}\right)^{n}$. The most general paper is [25] (see also Section 14 in [34]), where the regularity of a solution $u$ is studied on some "curved cones" $\Gamma \subset \mathbb{R}_{+}^{n}$, which have a finite tangency order to the walls of $\overline{\mathbb{R}}_{+}^{n}$; the regularity is measured in terms of a Sobolev-like scale by using spaces of Mellin distributions $M_{(\omega)}^{\prime, s}$, 
$\omega \in \mathbb{R}^{n}, s \in \mathbb{R}$ (the distributions in $M_{(\omega)}^{\prime, s_{1}}$ are more regular than those in $M_{(\omega)}^{\prime, s_{2}}$ if $\left.s_{1}<s_{2}\right)$. Suppose that $f \in M_{(\omega)}^{\prime, s} \Gamma$-locally at zero, i.e., for some cut-off function $\kappa$ subordinate to $\Gamma, \kappa f \in M_{(\omega)}^{\prime, s}$. The main result states that there exists $v \in M_{(\omega)}^{\prime, s-m} \Gamma$-locally such that $R v=f$ in the vicinity of zero in $\Gamma$. In particular, the authors obtained results on 2-local regularity of solutions at zero, in the direction $\delta x \in \mathbb{R}_{+}^{n}$ (see [R1]).

Ziemian's observation that the Mellin transformation is well adapted to describe microlocal singularities of distributions resulted in a joint paper [28] with Henryk Kołakowski, in which a simple proof of the crucial theorem of J. M. Bony on the propagation of 2-microlocal singularities was presented. The power of the Mellin transformation method was also demonstrated in the study of elliptic corner operators $R(x, x \partial / \partial x)$ in $[29,32,33]$. It turns out that solutions to $R u=0$ do not, in general, expand into discrete powers of the radial variable, but have continuous radial expansions whose densities are distributions supported by the radial characteristic set. In dimension $n=2$, the radial characteristic set consists of several half lines, and the densities can be explicitly computed ([33]; see also [R1]).

The fundamental research on the Mellin transformation and regularity of solutions to Fuchsian equations resulted in the monograph [34] by B. Ziemian and Z. Szmydt. The book provides a systematic treatment of multidimensional (local) Mellin transformation with its relations to the Fourier-Mellin, Fourier-Laplace, Cauchy, and Hilbert transformations, including the study of multipliers, and Paley-Wiener type theorems. However, the core of the book is the study of radial regularity of solutions to linear Fuchsian PDEs, i.e., equations of the form $R(x, x \partial / \partial x) u=f$, where $R(x, z)$ is a polynomial in $z$ with smooth (analytic) coefficients. In the Appendix written by Ziemian (see also [27]), connections between the theories of the Mellin, Borel, and Cauchy transformations, and the theories of generalized smooth functions and resurgent functions of J. Ecalle are established. It is worth noting that the theory of generalized Taylor expansions provides a natural interpretation of the Borel transformation.

One of Ziemian's most fruitful ideas seems to be the concept of a generalized analytic function (GAF for short), which appeared for the first time in [34] (see also [R2]). To explain its origins, let us observe that in the Taylor decomposition $(*)$ of a function $u$ analytic at zero, one can pass with $r$ to infinity. Thus,

$$
u(x)=S\left[x^{\alpha}\right] \quad \text { for } 0<x<\varrho,
$$

where $S=\sum_{i=0}^{\infty} a_{i} \delta_{(i)}$. The idea of the generalization is based on taking for $S$ a functional from a class of functionals acting on functions $\alpha \mapsto x^{\alpha}$. It appears that the most important class here is the class of Laplace distributions on $\overline{\mathbb{R}}_{+}$, which can be represented as sums of derivatives of continuous 
functions on $\overline{\mathbb{R}}_{+}$with at most exponential growth at infinity. The above idea led B. Ziemian to the definition of a GAF:

Definition. A function $u:(0, \varrho) \rightarrow \mathbb{C}$ is called a generalized analytic function with radius of convergence $\varrho>0$ if for any $\kappa>0$ there are $m_{\kappa} \in \mathbb{N}$ and continuous functions $S_{k}, 0 \leq k \leq m_{\kappa}$, with support in $\overline{\mathbb{R}}_{+}$satisfying $\left|S_{k}(\alpha)\right| \leq C e^{\kappa \alpha} \varrho^{-\alpha}$ for $\alpha \geq 0,0 \leq k \leq m_{\kappa}$, and

$$
u(x)=\sum_{k=0}^{m_{\kappa}}(-\ln x)^{k} \int_{0}^{\infty} S_{k}(\alpha) x^{\alpha} d \alpha \quad \text { for } 0<x<\varrho e^{-\kappa} .
$$

The definition of GAFs extends to the $n$-dimensional case by taking for $S$ a Laplace distribution supported by $\overline{\mathbb{R}}_{+}^{n}$, or more generally, its image under a complex mapping. The theory of GAFs was systematically developed in the important paper [39], in the broad context of functional and complex analysis, and its relations to the theory of resurgent functions. It turns out that many important special functions as well as solutions to some classes of linear singular ordinary and partial differential equations are GAFs (see [R2]).

Bogdan Ziemian derived (in [35]) new integral representations for fundamental solutions to a class of constant-coefficient operators (see [R3]). The formulas involve integrations over $(n-1)$-dimensional sets $\Gamma_{j}$ homeomorphic to $\overline{\mathbb{R}}_{+}^{n-1}$ which are contained in the complex characteristic set char $P=\left\{z \in \mathbb{C}^{n}: P(z)=0\right\}$, and can be regarded as $(n-1)$-dimensional Laplace type integrals. They can be considered as a generalization of Ehrenpreis formulas describing the behaviour of solutions at infinity, as well as a generalization of the Leray residue formula to the case where the cycle intersects the singular set of the integrand. Their most important new feature is an explicit expression of the asymptotic behaviour of the fundamental solution at infinity, which can be viewed as a multidimensional Borel resummation at infinity. The possibitity of deforming the sets $\Gamma_{j}$ within the complex characteristic set exhibits another phenomenon called by Ziemian the coupled resurgence effect. This in turn leads to the expectation that the new formulas may be useful in the study of nonlinear equations. In fact, Ziemian extended the resummation technique of J. Ecalle and W. B. J. Braaksma, and applied it to the semilinear Laplace equation $\Delta u=f(x, u)$ in two variables in [42] (jointly with M. E. Pliś).

The papers [35, 39] stimulated his joint work with Z. Szmydt on the next monograph Laplace Distributions in One and Several Variables. The book was intended to present in an accessible way some results of [35, 39], and to develop a mathematical apparatus required for Ziemian's plans to study nonlinear PDEs. In particular, he planned to devote a chapter to resurgent distributions and hyperfunctions, with a special attention paid to the Martineau-Harvey and Paley-Wiener type theorems. Parts of this book 
appeared in $[43,44]$ and in [P2]. In [43, 44], the theory of Laplace distributions is approached from the point of view of functional analysis and hyperfunction theory, which enables one to prove results of Martineau-Harvey type, establishing topological isomorphisms between spaces of Laplace distributions and some quotient spaces of holomorphic functions of exponential growth. These results lead to the topological imbedding of Laplace distributions into Laplace hyperfunctions. In [P2], Ziemian introduced the concept of holomorphic regularizations of meromorphic functions, which leads to the definition of resurgent hyperfunctions on singular complex spaces with normal crossing.

B. Ziemian posed many open problems (see [35] and his research project, cf. [P3]). He also bequeathed numerous ideas on methods which he intended to elaborate, and whose efficiency in PDEs he expected (cf. [P2], [P3]). Unfortunately, many of these ideas have now been lost to us.

Bogdan Ziemian was also very keen to play an active role in the promotion and development of Polish mathematics. He undertook several important duties for PAS: he was the secretary of the PAS Committee for Scientific Research and of the Scientific Council of Stefan Banach International Mathematical Center; a member of the Scientific Councils of IM PAS and of the Juliusz Schauder Center for Nonlinear Studies in Torun, Poland; head of the Section of Differential Equations of IM PAS; finally, he was appointed deputy director of IM PAS in 1995. He was also a member of the editorial boards of the proceedings series "Banach Center Publications" and of the journal "Annales Polonici Mathematici".

He was twice granted the Award of the Scientific Secretary of PAS, in 1986 and 1989.

The wide spectrum of the mathematical research of B. Ziemian, the novelty of analytical and geometrical methods he applied, and the significance of his results were noticed and appreciated by many distinguished mathematical institutions and universities; he was invited by: Mittag-Leffler Institute in Stockholm, Mathematisches Forschungsinstitut in Oberwolfach, Uppsala University, Geneva University, Toronto University, San Francisco University, Cambridge University. He spent some time as a visiting professor in Tokyo University and the University of Tours.

B. Ziemian will also be remembered as an active organizer of conferences, workshops, and research meetings. He was a co-organizer of three Banach Center semesters: "Partial Differential Equations" in 1990, "Singularities and Differential Equations" in 1993, and "Differential Geometry and Mathematical Physics" in 1995. 
He was elected chairman of the Steering Committee of the Series of Conferences on Mathematical Analysis by the European Science Foundation (ESF). In 1995, he organized the conference "Local Singularities of Solutions to Nonlinear and Singular PDEs", which assembled leading mathematicians working in the field.

He was one of initiators of creating International Post-Graduate Studies at IM PAS for which he prepared a programme, jointly with R. Dwilewicz. As deputy director of the Institute he tried to prevent drainage of talented young mathematicians by establishing special research positions and bonuses.

He initiated new forms of all-Polish mathematical activity. The first was the Mathematical Symposia established jointly with S. Janeczko and B. Jakubczyk, which aimed at acquainting the mathematical community with the newest achievements in mathematics. The second was a CracowWarsaw seminar joining the Cracow group of analytical geometers of Professors S. Łojasiewicz and T. Winiarski with the Warsaw group of researchers in the singularity theory of differential equations: B. Ziemian, B. Jakubczyk, S. Janeczko, T. Mostowski, H. Żołądek and their students. The third initiative was organization of workshops for young mathematicians, which were also attended by professors and invited speakers from abroad. He helped many talented young mathematicians to start collaboration with foreign specialists. He treated seriously popularization of the newest achievements in mathematics; he held well-prepared lectures for a general mathematical audience, and wrote an article [41] (jointly with G. Łysik) which clearly presents some new ideas concerning the theory of generalized analytic functions and its applications in differential equations.

He gave a series of lectures to post-graduate students of the Department of Mathematics of Warsaw University and IM PAS in 1991/92. They covered selected material ranging from regular and singular (semilinear) ordinary differential equations to partial differential equations of constant strength and singular partial differential equations (holonomic systems). The feature of the lectures was the use of the methods of complex analysis and the emphasis laid on the geometric character of the techniques and results. The lectures ([P1]) met with a vivid interest of students and researchers.

As a member of the editorial board of Annales Mathematici Polonici, he proposed, jointly with Roman Dwilewicz, some changes to make the journal more competitive. Since he treated the duties of a referee very seriously, many renowned publishers sought his opinion on books and mathematical papers.

For many years, B. Ziemian conducted a seminar "Generalized functions and differential equations" at IM PAS. He presented details of his theory of 
singular differential equations and pointed out new research projects. This resulted in joint papers, like those with Maria E. Pliś and Nguyen Si Minh; also a number of Grzegorz Łysik's papers developed the ideas introduced by Ziemian at the seminar.

Bogdan Ziemian was a man of science, but was also possessed of a warm humanity. The great services he rendered Polish mathematics lie not only in his mathematical legacy, but also in the generosity of spirit he extended to others. He had a particular gift for communicating ideas. He was always ready to give professional advice to whomever sought it. And his enthusiasm for mathematics was positively infectious to all who knew him. His peculiar gift for capturing the essence of problems allowed him to help other mathematicians, even those working in other fields. As such he was highly esteemed in the eyes of both professional colleagues and students.

Other aspects of Ziemian's personality were a strong spiritual and intellectual dimension. He spoke many foreign languages, and was deeply interested in philosophical problems as well as modern theoretical physics. He showed a great appreciation for the beauty of nature, reflected in his keen interest in photography. He practised sports (jogging and skiing) and was an avid mountaineer. Though gentle and unostentatious by nature, he could be both resolute and courageous in his actions. Assisting others was profoundly rooted in his nature.

On March 13, 1997, Bogdan and his wife were involved in a tragic accident. Bogdan Ziemian did not survive; his final act was to save the life of his wife.

\section{Bogdan Ziemian's publications}

[1] The group $G$ of proper Lorentz transformations and $G$-invariant distributions, Appendix in: Z. Szmydt, Fourier Transformations and Linear Differential Equations, PWN, Warszawa, and Reidel, Dordrecht, 1977, 464-471.

[2] On distributions invariant with respect to some linear transformations, Ann. Polon. Math. 36 (1979), 261-276.

[3] Fundamental solution $E_{n}$ of the operator $\partial^{2} / \partial t^{2}-\Delta_{n}$ for $n \geq 3$ (with Z. Szmydt), ibid., 277-286.

[4] On G-invariant distributions, J. Differential Equations 35 (1980), 66-86.

[5] Special solutions of the equations $P u=0, P u=\delta$ for invariant linear differential operators with polynomial coefficients (with Z. Szmydt), J. Differential Equations 39 (1981), 226-256. 
[6] Distributions invariant under compact Lie groups, Ann. Polon. Math. 42 (1983), 175-183.

[7] Fundamental solution for operators preserving a quadratic form (with Z. Szmydt), ibid., 369-386.

[8] A method for constructing invariant fundamental solutions for invariant operators (with Z. Szmydt), in: Proc. Conf. Convergence and Generalized Functions (Katowice, 1983), IM PAN, Warszawa, 1984, 149-155.

[9] A method for constructing invariant fundamental solutions for $P\left(\Delta_{m}\right)$ (with Z. Szmydt), Zeszyty Naukowe Politechniki Śląskiej Ser. Mat.-Fiz. 48 (1986), 147-164.

[10] Explicit invariant solutions for invariant linear differential operators (with Z. Szmydt), Proc. Roy. Soc. Edinburgh 98 (1984), 149-166.

[11] Local order function for homogeneous rotation invariant distributions and their multiplication (with Z. Szmydt), Ann. Univ. Mariae Curie-Skłodowska Sect. A 38 (1984), 139-143.

[12] A Taylor type decomposition for distributions in one dimension, Bull. Polish Acad. Sci. Math. 32 (1984), 143-155.

[13] An analysis of microlocal singularities of functions and distributions on the real line, ibid., 157-164.

[14] The derivative of a measurable function and of a distribution at a point and its basic properties, ibid., 165-177.

[15] An invariance method for constructing fundamental solutions for $P\left(\square_{m n}\right)$ (with Z. Szmydt), Ann. Polon. Math. 46 (1985), 333-360.

[16] Singular ordinary differential equations on spaces of singular test functions with applications to invariant partial differential operators (with Z. Szmydt), in: Proc. Conf. Differential Equations and Applications (Rousse, 1985), 1987, 955-958.

[17] The Mellin transformation and microlocal singularities of distributions, ibid., 10051008.

[18] Invariant fundamental solution of the wave operator (with Z. Szmydt), Demonstratio Math. 19 (1986), 371-386.

[19] Taylor formula for distributions in several dimensions, Bull. Polish Acad. Sci. Math. 34 (1986), 277-286.

[20] Multidimensional Mellin transformation and partial differential operators with regular singularity (with Z. Szmydt), ibid. 35 (1987), 167-180.

[21] Mellin analysis of singularities (with G. Łysik), in: Proc. Internat. Summer School on Nonlinear Differential Equations (Varna, 1987).

[22] Solutions of singular elliptic equations via the Mellin transformation on sets of high order of tangency to the singular lines (with Z. Szmydt), Bull. Polish Acad. Sci. Math. 36 (1988), 521-535.

[23] Taylor formula for distributions, Dissertationes Math. (Rozprawy Mat.) 264 (1988).

[24] The Mellin transformation and multidimensional generalized Taylor expansions of singular functions, J. Fac. Sci. Univ. Tokyo Sect. IA Math. 36 (1989), 263-295.

[25] Local existence and regularity of solutions of singular elliptic operators on manifolds with corner singularities (with Z. Szmydt), J. Differential Equations 83 (1990), $1-25$.

[26] Mean value theorems for linear and semi-linear rotation invariant operators, Ann. Polon. Math. 51 (1990), 341-348.

[27] Generalized Taylor expansions and theory of resurgent functions of Jean Ecalle, in: Proc. Conf. Generalized Functions and Convergence (Katowice, 1988), World Sci., 1990, 285-295. 
[28] Second microlocalization and the Mellin transformation (with H. Kolakowski), Publ. Res. Inst. Math. Sci. 26 (1990), 785-801.

[29] Continuous radial asymptotics for solutions to elliptic Fuchsian equations in 2dimensions, in: Proc. Sympos. Microlocal Analysis and Its Applications (Kyoto, 1990), Sûrikaisekikenkyûsho Kôkyûroku 750, Kyoto, 1991, 3-19.

[30] The modified Cauchy transformation with applications to generalized Taylor expansions, Studia Math. 102 (1992), 1-24.

[31] Characterization of Mellin distributions supported by certain noncompact sets (with Z. Szmydt), ibid., 25-38.

[32] Elliptic corner operators in spaces with continuous radial asymptotics I, J. Differential Equations 101 (1993), 28-57.

[33] Elliptic corner operators in spaces with continuous radial asymptotics II, in: Partial Differential Equations, Banach Center Publ. 27, IM PAN, Warszawa, 1992, 555-580.

[34] The Mellin Transformation and Fuchsian Type Partial Differential Equations (with Z. Szmydt), Math. Appl. 56, Kluwer, Dordrecht, 1992.

[35] Leray residue formula and asymptotics of solutions to constant coefficient PDEs, Topol. Methods Nonlinear Anal. 3 (1994), 257-293.

[36] Mellin analysis of singular and non-linear PDEs, in: Proc. Conf. Equadiff 8 (Bratislava, 1993), Tatra Mt. Math. Publ. 4 (1994), 243-248.

[37] Exact radial asymptotics of solutions to singular elliptic differential equations (with G. Eysik), in: Proc. Fifth Internat. Colloquium on Differential Equations (Plovdiv, 1994), VSP, Utrecht, 1995, 213-221.

[38] Between the Paley-Wiener theorem and the Bochner tube theorem (with Z. Szmydt), Ann. Polon. Math. 60 (1995), 299-304.

[39] Generalized analytic functions with applications to singular ordinary and partial differential equations, Dissertationes Math. (Rozprawy Mat.) 354 (1996).

[40] A remark on Nilsson type integrals (with Nguyen Si Minh), in: Singularities and Differential Equations, Banach Center Publ. 33, IM PAN, Warszawa, 1996, 277-285.

[41] Uogólnione funkcje analityczne z zastosowaniami [Generalized analytic functions with applications] (with G. Łysik), Wiadom. Mat. 32 (1996), 15-25 (in Polish).

[42] Borel resummation of formal solutions to nonlinear Laplace equations in 2 variables (with M. E. Pliś), Ann. Polon. Math. 67 (1997), 31-41.

[43] Laplace distributions and hyperfunctions on $\overline{\mathbb{R}}_{+}^{n}$ (with Z. Szmydt), J. Math. Sci. Tokyo Univ. 5 (1998), 41-74.

[44] Topological imbedding of Laplace distributions in Laplace hyperfunctions (with Z. Szmydt), Dissertationes Math. (Rozprawy Mat.) 376 (1998).

[45] Convolution equations in the space of Laplace distributions (with M. E. Pliś), Ann. Polon. Math. 69 (1998), 271-181.

[46] On extendability of invariant distributions, posthumous, this volume, 13-25.

\section{Bogdan Ziemian's preprints}

[P1] 20 lectures on ordinary and partial differential equations. Geometric methods of complex analysis, preprint, IM PAN, Warszawa, 1992.

[P2] Holomorphic regularization of meromorphic functions, preprint, Université de Tours, 1996.

[P3] Unfinished notes of Bogdan Ziemian, preprint, IM PAN, Warszawa, 2000. 


\section{Other references}

[R1] H. Kołakowski, Mellin analysis of partial differential equations in papers of B. Ziemian, this volume, 27-33.

[R2] G. Łysik, Generalized analytic functions of Bogdan Ziemian, this volume, 35-41.

[R3] - Laplace integrals in partial differential equations in papers of Bogdan Ziemian, this volume, 43-50. 